\title{
Silicotuberculosis in the elderly: report of two cases
}

\author{
F. Scafa1, C.M. Minelli2, R. Fonte'2, G.L. Rosso², \\ M.I. Cappelli2, S.M. Candura²
}

ABSTRACT: Silicotuberculosis in the elderly: report of two cases. F. Scafa, C.M. Minelli, R. Fonte, G.L. Rosso, M.I. Cappelli, S.M. Candura.

Silicotuberculosis is observed rarely in the current clinical practice. We present two patients (a 72-year-old man and a 84-year-old woman) who developed silicosis after having worked for several decades in the ceramics industry. In both, pulmonary tuberculosis complicated the clinical picture several years after retirement. The first subject presented a multicavitary lesion in the apex of the right lung, which subsequently evolved with fibrosis. The other developed bilateral tubercular bronchopneumonia and right tubercular pleurisy, that improved after pro- longed antimycobacterial polychemotherapy. The two cases confirm that patients with silicosis are at an increased risk of developing tuberculosis, and show that, nowadays, silicotuberculosis may represent a geriatric problem. In the elderly, recognition of tuberculosis associated with silicosis is often difficult. Occupational history, radiology (conventional chest radiography and computed tomography) and microbiology (identification of Mycobacterium tuberculosis in sputum and pleural exudate) are helpful for the correct diagnosis, which, in turn, is important for prognosis and treatment, as well as in relation to medico-legal issues and occupational-related compensation claims.

Monaldi Arch Chest Dis 2004; 61: 4, 241-243.

Keywords: Silicosis, tuberculosis, occupational disease, ceramics industry, radiodiagnostics.

Unità Operative di Medicina del Lavoro, Istituti Scientifici di Tradate 1 \& di Pavia ${ }^{2}$, Scuola di Specializzazione in Medicina del Lavoro, Università degli Studi di Pavia; Fondazione Salvatore Maugeri, Clinica del Lavoro e della Riabilitazione, IRCCS - Italy.

Correspondence: Prof. Stefano M. Candura, UO di Medicina del Lavoro, Fondazione Salvatore Maugeri, via Ferrata 8, I-27100 Pavia-Italy; e-mail: scandura@fsm.it

\section{Introduction}

Silicosis is an occupational lung disease caused by the inhalation and interstitial deposition of crystalline silica $\left(\mathrm{SiO}_{2}\right)[1]$. Occupations at risk include mining and milling, tunneling, foundry work, building/demolition, sandblasting, and production of siliceous materials (glass, ceramics, abrasives, refractories) [2, 3].

Pulmonary tuberculosis has been for decades a frequent and feared complication of silicosis [2,4]. Nowadays, thanks to the improvement of workplace hygienic conditions, reports of new cases of silicosis are progressively decreasing, in Italy [5, $6]$ as well as in other Western Countries [2, 7]. Since the advent of effective drug therapies, the prevalence of tuberculosis has also declined (although a recrudescence is currently being observed) $[8,9]$. As a result, silicotuberculosis is encountered rarely in the current clinical practice. Here we report the clinical and radiological findings of two cases disclosed in advanced age.

\section{Case reports}

The first patient is a 72-year-old man, who had been employed in a ceramic bathtub factory from the age 33 to the age 55 , when he retired. His duties included mixing and milling of first mat- ters, foundry work and sandblasting, exposing him to the inhalation of crystalline silica and other dusts. In 1996, an occasional chest radiography revealed diffuse, bilateral, partially confluent lung micronodules. These findings were confirmed by high resolution computed tomography (HRCT), which additionally showed a multicavitary conglomeration in the apex of the right lung. Tuberculin skin testing was positive. Mycobacteria were not found in the sputum. Although a clear diagnosis of silicotuberculosis had not been formulated at the time, the case was referred to the Italian Workers' Compensation Authority (INAIL) which classified the disease as "silicosis" and recognised its occupational origin. Recently, the patient filed a new occupational-related compensation claim, asking for a new medico-legal evaluation.

The subject came under our observation on April 2002 complaining of a worsening of the general conditions and dyspnea occurring after mild physical exertion. Chest X-ray and HRCT demonstrated interstitial fibrosis with bilateral micronodular dissemination and diffuse emphysema. Foci of pleural fibrosis were present on the right. The apical opacity described in 1996 was enlarged and increased in density (figure 1), suggesting fibrocalcific organization of the previous multicavitary lesion. Spirometry showed a restrictive pattern 


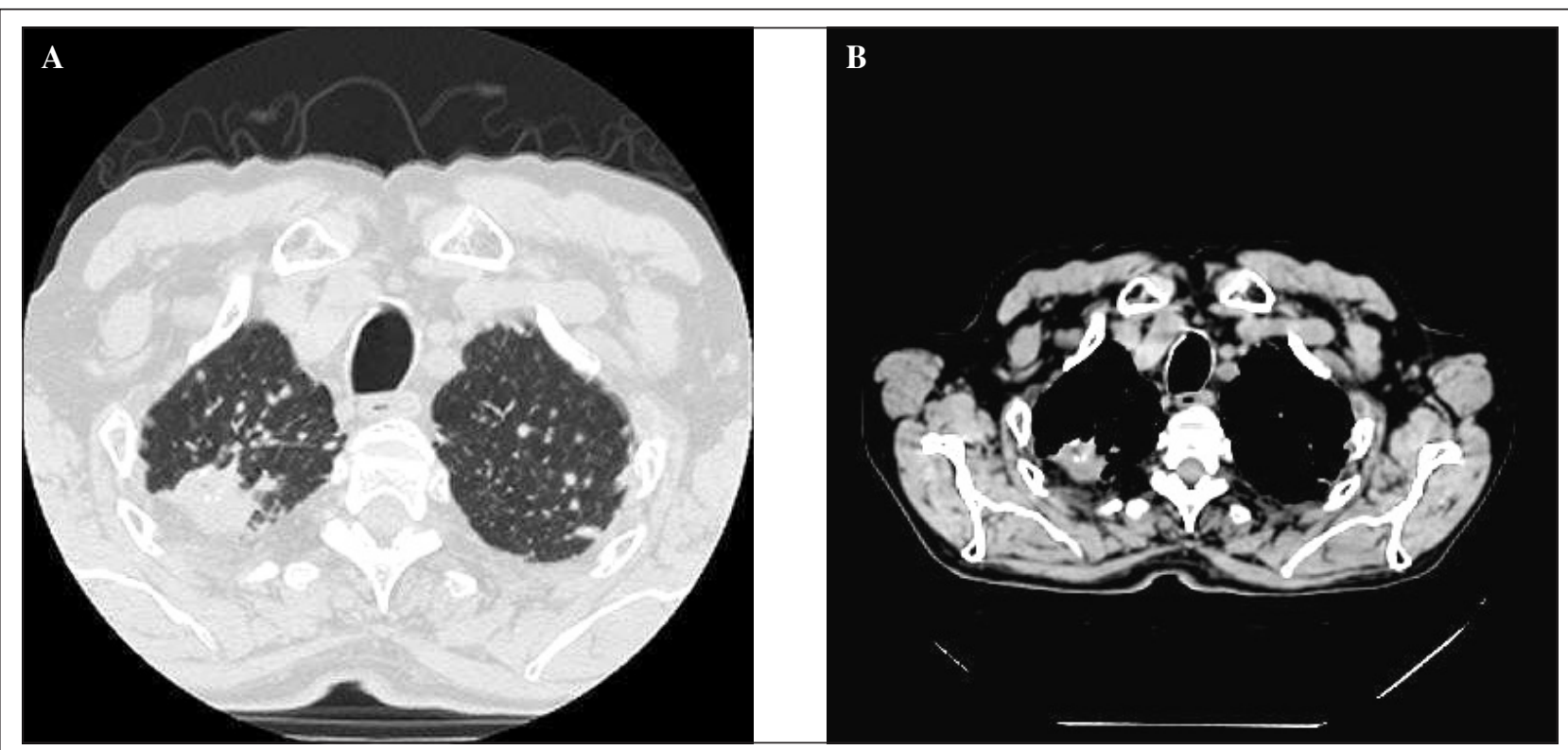

Fig. 1. - A: Chest computed tomography showing a large, disomogeneous opacity in the apical posterior segment of the right lung. B: The same image at higher resolution.

with a $33 \%$ decrease in the forced vital capacity (FVC). Final diagnosis was: severe silicosis complicated by tuberculosis (evoluted with fibrosis) of the right upper lobe; ILO (International Labour Office) classification: q 3/3; tb, em, pl, co. The medico-legal revaluation for compensation is still ongoing.

The second patient (a 84-year-old woman) was heavily exposed to crystalline silica from age 14 to age 55 (year of retirement) in various departments (mixing and milling, moulding, drying and baking, finishing and decoration) of a factory that produced ceramics for industrial or ornamental use (e.g., insulators, pottery). Silicosis was diagnosed during the last year of employement, and the occupational origin of the disease was recognised by INAIL.

On January 2003 the patient was hospitalised for persistent fever $\left(\sim 38^{\circ} \mathrm{C}\right)$. Chest examination revealed dullness and decreased breath sound over the right pulmonary base. The erythrocyte sedimentation rate $(109 \mathrm{~mm} / \mathrm{h})$ and reactive protein $\mathrm{C}$ concentration $(10.10 \mathrm{U} / \mathrm{L})$ were increased. Chest radiography showed scattered, partially confluent, parenchimal opacities in the lower half of the right lung, associated with omolateral pleural effusion and diffuse interstitial alteration. Small consolidations were also present in the parahilar area of the left lung. These findings were confirmed by computed tomography that additionally demonstrated a triangular, heterogeneous, partially calcific opacity in the posterior segment of the right upper lobe. Mycobacterium tuberculosis was isolated in both sputum and pleural exudate cultures. Diagnosis was: bilateral tubercular bronchopneumonia and right tubercular pleurisy complicating silicosis. Sensitivity test-based antitubercular polychemotherapy (rifampicin, pyrazinamide, isoniazid, ethambutol) led to a gradual improvement of the clinical picture. Currently (January 2004), the patient is feeling reasonably well. Her body temperature is normal. Chemotherapy and follow-up are still ongoing. In the meanwhile, the case has been referred to INAIL for medico-legal revaluation.

\section{Discussion}

While confirming that patients with silicosis are at increased risk of developing pulmonary tuberculosis, the cases presented here indicate that, nowadays, silicotuberculosis may represent a geriatric problem. Indeed, the risk of developing tuberculosis increases with the severity of pre-existing silicosis $[10,11]$. In comparison with the past, silicosis is usually diagnosed later in life and in a less evoluted stage $[2,5]$. Consequently, silicotuberculosis may require a longer time to develop, and therefore arrive to medical observation in the elderly, usually (as in the two patients described) several years after retirement.

Silica exposure leads to impaired macrophage function and cell-mediated immunity. Alterations in lymphocyte subsets (reduced number of T cells and increased number of $B$ cells) and immunoglobulins levels have been observed in patients with silicosis [1]. Experimental studies have shown an association between changes induced by silica particles and increased susceptibility to mycobacterial infection [12, 13], whereas epidemiological data indicate that occupational exposure to silica alone, in the absence of silicosis, is dose-dependently associated with increased risk of tuberculosis, even after the exposure ends [14-16]. Thus, pulmonologists, occupational physicians and geriatrists should be aware of the possibilty of tuberculosis in subjects with an history of silica dust exposure, especially considering that the tuberculosis decline observed in the industrialized countries over the past decades has recently lev- 
elled off or reversed in both Europe and the USA $[8,9]$.

In the elderly, recognition of tuberculosis complicating silicosis is often difficult. Symptomatology (cough, dyspnea, fever, night sweats, general malaise, fatigability) and laboratory findings (e.g., accelerated sedimentation rate) are aspecific, and may be due to other conditions, such as bronchitis, non-tubercular infections, malnutrition, or a worsening of the silicosis. Additionally, sputum smears and cultures for mycobacteria may conclude in a falsely negative result, presumably because the bacilli are "walled-off" by the silicotic fibrosis and, therefore, are not discharged into the bronchi [4].

Diagnostic doubts can be resolved by the complementary utilisation of conventional radiography and computed tomography. Findings suggestive for silicotuberculosis include the coalescence of pre-existing silicotic nodules and/or the rapid appearance of new opacities (especially in the apical posterior segments of the upper lobes and in the superior segments of the lower lobes), as well as the development of pleural or pericardial effusion. Cavities are the most important radiologic signs of activity and infectivity. In silicotic patients, they usually appear within a conglomeration of nodules, and present irregularly shaped inner walls $[17,18]$.

Beside decreasing the sensitivity of microbiological diagnostic tests, the "walling-off" of mycobacteria by the silicotic fibrotic process hinders the penetration of antitubercular drugs. As a result, coexistent silicosis interferes with successful treatment of tuberculosis [4]. In the elderly, the response to pharmacological treatment may be further modified by age-related variations in drug metabolism and disposition $[19,20]$. As in the female patient described above, these problems may be partly overcome by prolonged polychemotherapy including rifampicin and pyrazinamide [21].

A discussion of the association between silicosis and tuberculosis is not complete without some mention of medico-legal considerations. Silicosis is an occupational disease and, as such, it has to be reported to the judicial and workers' compensation authorities. In silicotic patients, tuberculosis should be considered to be causally linked to previous silica dust inhalation in the work environment, even when it develops in the elderly, several years after the exposure has ceased. Thus, disclosure of silicotuberculosis always asks for medicolegal revaluation. The clinician may greatly facilitate this process (which is often long and troubled, as shown by the cases reported) through an accurate diagnostic and prognostic assessment of the disease, and the production of congruous medical documentation utilisable in the forensic setting.

\section{References}

1. Ding M, Chen F, Shi X, Yucesoy B, Mossman B, Vallyathan V. Diseases caused by silica: mechanisms of injury and disease development. Int Immunopharmacol 2002; 2: 173-182.

2. Rosenman KD, Reilly MJ, Kalinowski DJ, Watt FC. Silicosis in the 1990s. Chest 1997; 111: 779-786.

3. Candura F, Candura SM. Principles of Industrial Technology for Occupational Medicine Scholars' Use. Piacenza (Italy): CELT, 2002.

4. Snider DE. The relationship between tuberculosis and silicosis. Am Rev Resp Dis 1978; 118: 455-460.

5. Edallo A, Fonte R, Moscato G, Biscaldi G, Candura SM, Peressini S. Trends of occupational pathology in a clinical survey. G Ital Med Lav Erg 2001; 23: 407-408.

6. Italian Workers' Compensation Authority (INAIL) Annual Report 2001. Rome: INAIL, 2002.

7. Cherry NM, Meyer JD, Holt DL, Chen Y, McDonald JC. Surveillance of work-related diseases by occupational physicians in the UK: OPRA 1996-1999. Oсcup Med 2000; 50: 496-503.

8. Raviglione MC, Snider DE Jr, Kochi A. Global epidemiology of tuberculosis: morbidity and mortality of a world wide epidemic. JAMA 1995; 273: 220-226.

9. Migliori GB, Ambrosetti M. Epidemiology of tuberculosis in Europe. Monaldi Arch Chest Dis 1998; 53: 681-687.

10. Saiyed HN, Ghodasara NB, Sathwara NG, Patel GC, Parikh DJ, Kashyap SK. Dustiness, silicosis \& tuberculosis in small scale pottery workers. Indian J Med Res 1995; 102: 138-142.

11. Solomon A, Rees D, Felix M, Venter E. Silicosis and tuberculosis: a proposed radiographic classification of tuberculosis to accompany the ILO international classification of radiographs of pneumoconioses. Int J Occup Environ Health 2000; 6: 215-219.

12. Gross P, Westrick ML, McNerney JM. Experimental tuberculosis. Am Rev Resp Dis 1961; 83: 510.

13. Allison AC, D'Arcy Hart P. Potentiation of silica of the growth of Mycobacterium tuberculosis in macrophage cultures. Br J Exp Pathol 1968; 49: 465.

14. Sherson D, Lander F. Morbidity of pulmonary tuberculosis among silicotic and nonsilicotic foundry workers in Denmark. J Occup Med 1990; 32: 110-113.

15. Chen GX, Burnett CA, Cameron LL, et al. Tuberculosis mortality and silica exposure: a case-control study based on national mortality database for the years 19831992. Int J Occup Environ Health 1997; 3: 163-170.

16. Hnizdo E, Murray J. Risk of pulmonary tuberculosis relative to silicosis and exposure to silica dust in South African gold miners. Occup Environ Med 1998; 55: 496-502.

17. Kuhlman JE, Deutsch JH, Fishman EK, Siegelman SS. CT features of thoracic mycobacterial disease. Radiographics 1990; 10: 413-431.

18. Solomon A. Silicosis and tuberculosis: Part 2 A radiographic presentation of nodular tuberculosis and silicosis. Int J Occup Environ Health 2001; 7: 54-57.

19. Loi C-M, Vestal RE. Drug metabolism in the elderly. Pharmacol Ther 1988; 36: 131-149.

20. Dawling S, Crome P. Clinical pharmacokinetic considerations in the elderly: An update. Clin Pharmacokin 1989; 17: 236-263.

21. Bass JB, Farer LS, Hopewell PC, et al. Treatment of tuberculosis and tuberculosis infection in adults and children. Am J Respir Crit Care Med 1994; 149: 1359-1374. 